\title{
Gastric wall abscess after endoscopic submucosal dissection
}

\author{
Seung Jung Yu, Sang Heon Lee, Jun Sik Yoon, Hong Sub Lee, Sam Ryong Jee \\ Department of Internal Medicine, Inje University Busan Paik Hospital, Inje University College of Medicine, Busan, Korea
}

Gastric wall abscess, a localized form of phlegmonous gastritis, is a rare complication of endoscopic resection. We report the first case of gastric wall abscess developing after endoscopic submucosal dissection in Korea. A 72-year-old woman visited our clinic to receive treatment for gastric adenoma. The patient successfully underwent endoscopic submucosal dissection with no complications. The final diagnosis was well-differentiated tubular adenocarcinoma. We performed follow-up endoscopy 10 weeks later and found a large subepithelial lesion on the posterior wall of the gastric antrum. Abdominal computed tomography revealed hypodense wall thickening and a $5 \mathrm{~cm}$ heterogenous multilobular mass in the submucosal layer of the gastric antrum. Submucosal invasion with mucin-producing adenocarcinomas could therefore not be excluded. The patient agreed to undergo additional gastrectomy due to the possibility of a highly malignant lesion. The final diagnosis was acute suppurative inflammation with the formation of multiple abscesses in the mural layers and omentum. The patient was discharged with no complications.

Keywords: Early gastric cancer; Endoscopic submucosal resection; Gastric wall abscess; Phlegmonous gastritis

\section{INTRODUCTION}

Gastric endoscopic submucosal dissection (ESD) is a standard treatment for early gastric cancer (EGC) and gastric adenoma. Complications of this procedure include pain, bleeding, and perforation. Pain after resection is typically mild. Bleeding is the most common complication, and occurs in up to $7 \%$ of patients undergoing ESD. The risk of perforation in these patients is approximately $4 \% .{ }^{1}$ Phlegmonous gastritis, especially the formation of a localized abscess, is a rare complication associated with ESD. ${ }^{2}$ To the best of our knowledge, this is the first report on localized gastric wall abscess after gastric ESD for EGC in Korea.

Received: July 13, $2021 \quad$ Revised: September 2, 2021

Accepted: September 8, 2021

Correspondence: Sang Heon Lee

Department of Internal Medicine, Inje University Busan Paik Hospital, Inje University College of Medicine, 75 Bokji-ro, Busanjin-gu, Busan 47392, Korea E-mail: daschu94@naver.com

(a) This is an Open Access article distributed under the terms of the Creative Commons Attribution Non-Commercial License (http://creativecommons.org/licenses/by-nc/4.0/) which permits unrestricted non-commercial use, distribution, and reproduction in any medium, provided the original work is properly cited.

\section{CASE REPORT}

A 72-year-old woman visited the Busan Paik Hospital to receive treatment for gastric adenoma, which was discovered during screening esophagogastroduodenoscopy (EGD) at a local clinic. The patient's medical history was unremarkable aside from a 10-year history of hypertension. The patient was admitted for gastric ESD 2 weeks after initial EGD. The results of all initial laboratory tests performed at our hospital were within the normal range. The lesion was successfully excised using ESD on the day after admission (Fig. 1). The procedure was performed using a therapeutic endoscope (GIF-Q260J; Olympus Medical Systems, Tokyo, Japan). An initial mucosal incision and submucosal dissection were performed using a 2.0-mm DualKnife J (KD655-L; Olympus Medical Systems) after local injection of a sodium hyaluronate solution. Hemostasis for procedural bleeding was attempted using hemostatic forceps (FD-410LR; Olympus Medical Systems) in the soft coagulation mode (effect $4,70 \mathrm{~W}$ ). The dissected lesion was $1.2 \times 0.8 \mathrm{~cm}$ in size and had nodular ulcerated mucosa. Histopathological examination revealed well-differentiated tubular adenocarcinoma confined within the muscularis mucosa with no lymphovascular inva- 

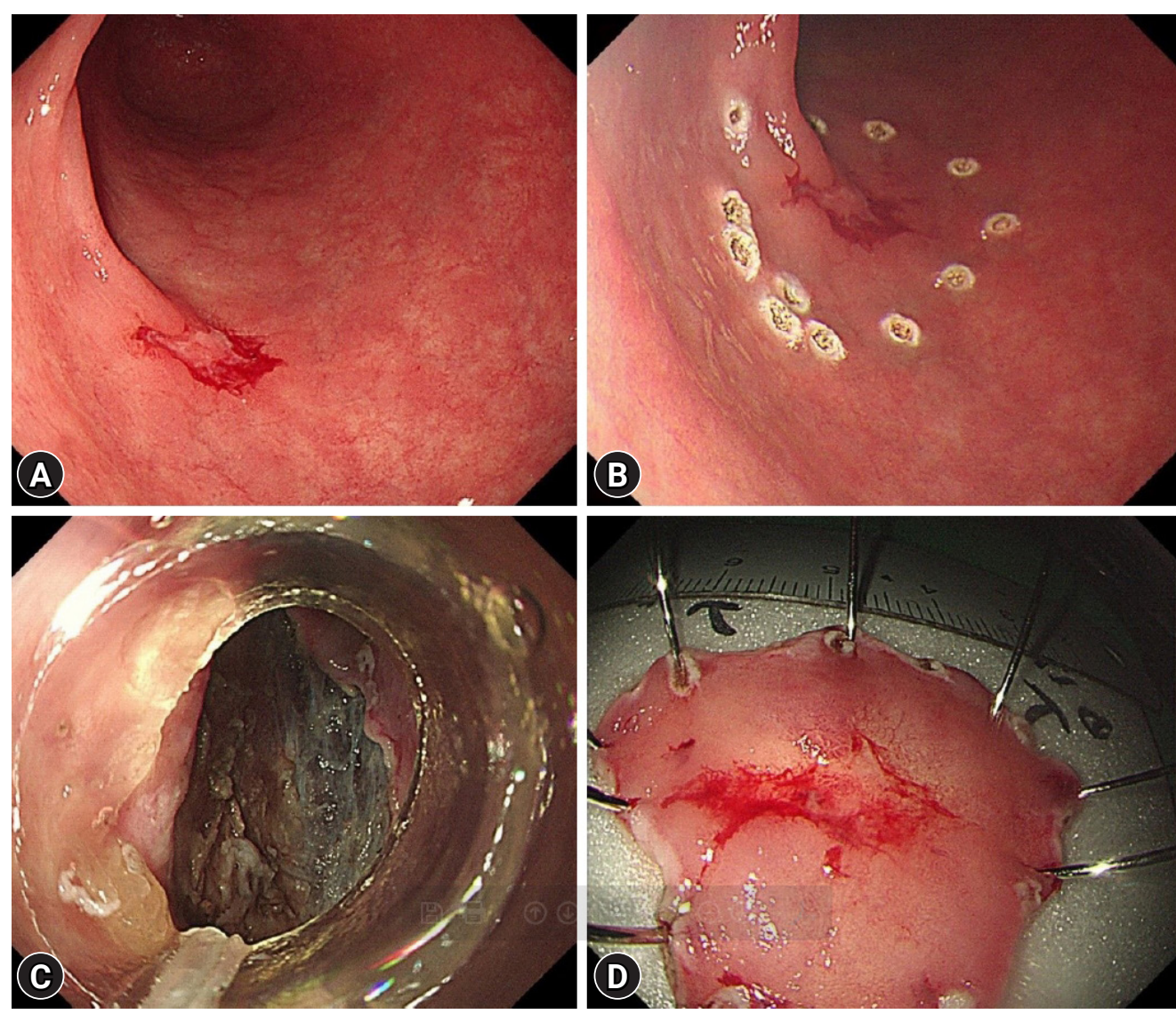

Fig. 1. Endoscopic submucosal dissection to remove the lesion. (A) An ulcerated lesion on the anterior wall side of the proximal antrum. (B) The lesion was marked using an endoscopic knife. (C) Dissection of the submucosal layer was performed. (D) A resected specimen.

sion. The resection margin was carcinoma-free. No free air was observed in the post-ESD X-ray. The patient was discharged the next day. The patient continued to take a proton pump inhibitor during hospitalization and during outpatient follow-up after discharge. She visited the clinic one week later for abdominal computed tomography (CT), and Helicobacter pylori eradication was initiated, during which a post-ESD ulcer lesion with no other masses or metastasis was revealed. Ten weeks after the procedure, she visited the clinic again for follow-up EGD. She exhibited no specific symptoms such as abdominal pain or fever. We observed a subepithelial lesion on the posterior wall of the gastric antrum, which was approximately $5 \mathrm{~cm}$ in diameter in the largest dimension (rolling sign, negative; cushion sign, positive) (Fig. 2). We suspected that this lesion might be a gastric abscess or submucosal invasion of the cancer. Thus, we used CT to ascertain the differential diagnosis. Abdominal CT showed hypodense wall thickening and a heterogeneous multilobular mass about $5 \mathrm{~cm}$ in size in the submucosal layer of the gastric antrum (Fig. 3), accompanied by local infiltration into the peripheral omentum. Endoscopic ultrasonography was not performed since it was thought that there was no further information besides the information obtained during CT. A possibility of a submucosal cancer was raised in the radiology department when previous CT scans were reviewed. We could not exclude submucosal tumor extension in mucin-producing cancers. Therefore, after obtaining the patient's agreement, we decided to proceed with surgical resection. The preoperative blood test rest results were as follows: white blood cell count, 10,180/ $\mu \mathrm{L}$ (percentage of neutrophils, 77.1\%); hemoglobin, 13.1 $\mathrm{g} / \mathrm{dL}$; platelet count, $437,000 / \mu \mathrm{L}$. The liver function test, blood urea nitrogen, creatinine, and electrolyte levels were all within normal limits. The C-reactive protein level was not tested. The patient underwent laparoscopic distal gastrectomy. Acute suppurative inflammation with multiple abscess formation in the mural layers and omentum was diagnosed based on pathological findings of the specimen (Fig. 4). She was discharged 7 days postoperatively with no complications and was regularly followed up in our outpatient clinic for 6 months, during which 

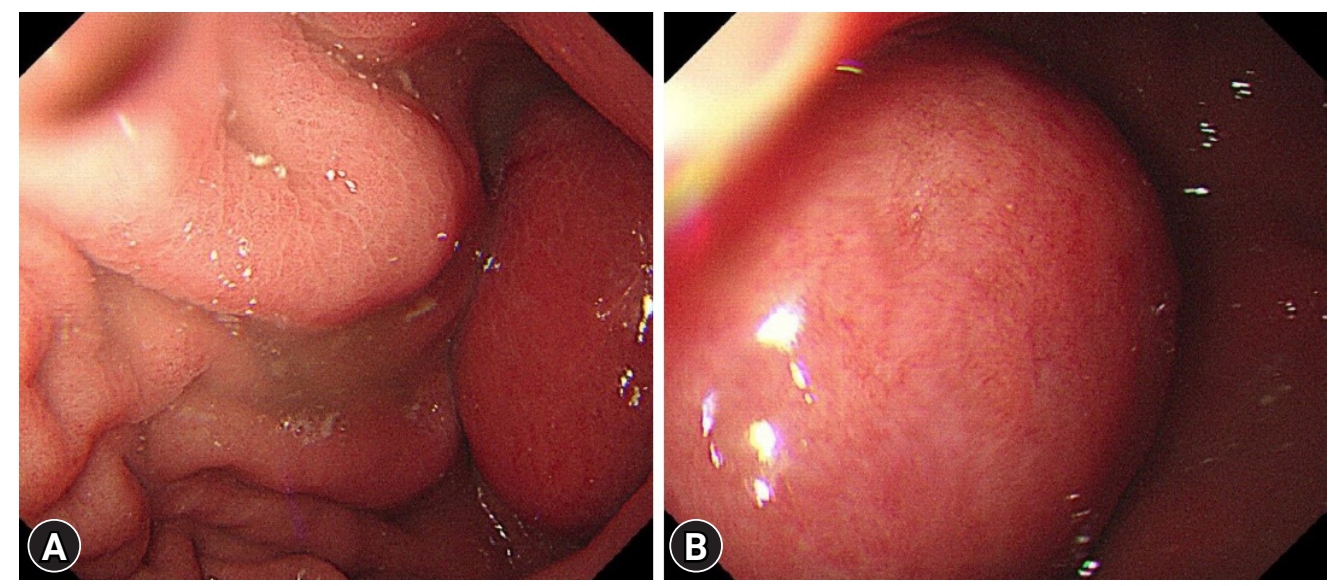

Fig. 2. (A, B) A 5-cm sized subepithelial lesion was observed on the posterior wall of the gastric antrum during the follow-up endoscopy.
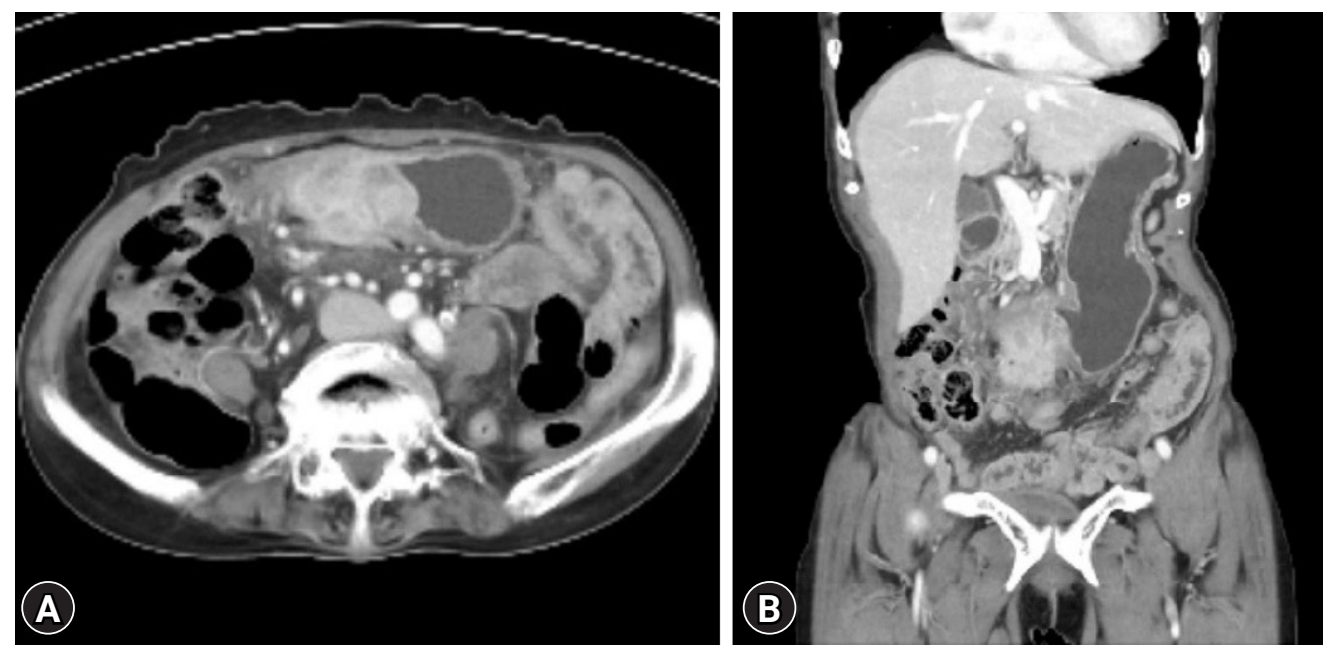

Fig. 3. (A, B) Computed tomography was performed after the subepithelial lesion was discovered. Hypodense wall thickening and a 5-cm sized heterogenous multilobular submucosal mass was observed in the gastric antrum. Local infiltration into the peripheral omentum was also observed.
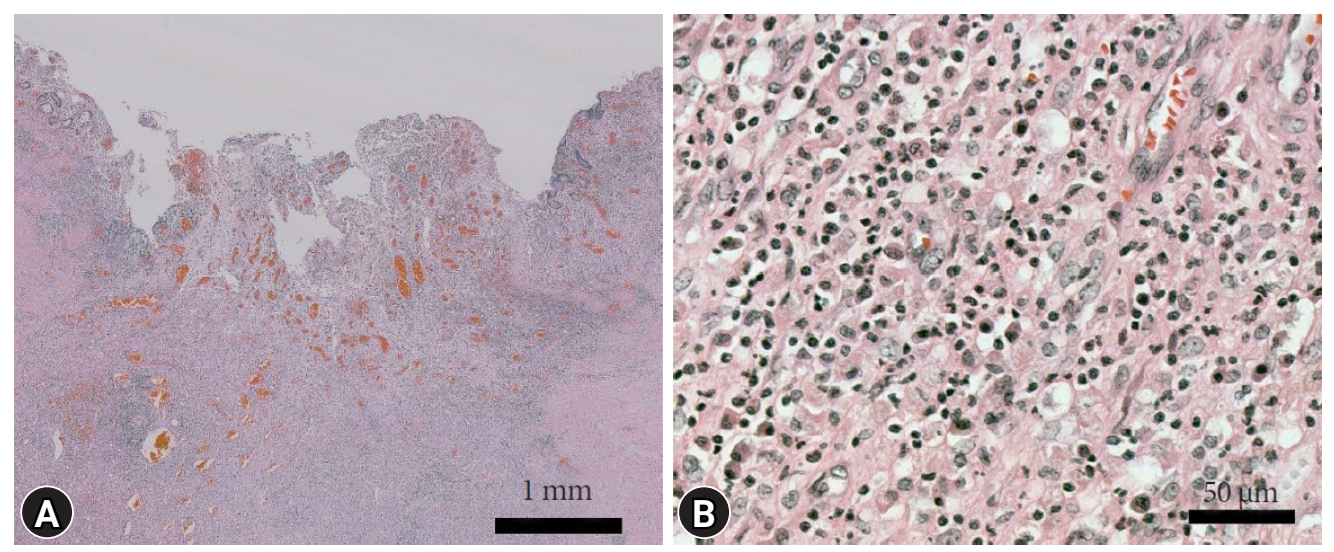

Fig. 4. Postoperative histological findings. (A, B) Acute suppurative gastritis with multifocal abscess formation and the infiltration of inflammatory cells in all layers of the stomach was seen (hematoxylin and eosin stain, $\times 15$ and $\times 200$ ). 
she showed no signs or symptoms of gastric abscess or EGC recurrence.

\section{DISCUSSION}

Phlegmonous gastritis is a rare form of acute gastritis usually caused by bacterial infection, and its mortality rate is approximately $50 \%$. The risk factors for phlegmonous gastritis include immunodeficiency, gastric carcinoma, prior therapeutic endoscopic procedure, oncologic disease, malnutrition, and chronic glucocorticoid use. However, healthy individuals make up approximately $50 \%$ of phlegmonous gastritis cases. Phlegmonous gastritis can be classified as one of two types depending on the extent of the disease: diffuse and localized. ${ }^{2,3}$ Gastric wall abscess is a localized form of phlegmonous gastritis.

Phlegmonous gastritis following ESD is a rare complication, and the simultaneous occurrence of localized phlegmonous gastritis and gastric wall abscess is extremely rare. ${ }^{4,5}$ To the best of our knowledge, only three cases of phlegmonous gastritis after $\mathrm{ESD}^{6-8}$ and one case of phlegmonous gastritis after endoscopic mucosal resection ${ }^{9}$ have been reported in the literature. Furthermore, three cases of perigastric abscesses after ESD have been reported worldwide. ${ }^{10-12}$ Perigastric abscesses are thought to be caused by the passage of bacteria through the gastric wall, so it is usually assumed that perforation has occurred or that an unidentified microperforation has formed. ${ }^{10}$ Although the pathophysiological mechanism differs from that of gastric abscess, we reviewed them together since both are infectious complications that may occur after endoscopic resection and the treatment is similar (Table 1$).^{6-12}$

Immunocompromised patients tend to be susceptible to phlegmonous gastritis after endoscopic procedures. Therefore, prophylactic antibiotics are recommended for immunosuppressed patients in some cases. ${ }^{6,8}$ However, our patient had no specific medical history besides hypertension. This is consistent with the literature, as two of the four reported cases (50\%) had no relevant medical history. Therefore, it is important to consider that phlegmonous gastritis may even develop in patients with healthy immune system.

Diffuse phlegmonous gastritis progresses rapidly and causes symptoms within 24 hours. On the other hand, gastric wall abscess and perigastric abscess take longer to develop (between 5 days and 10 weeks). It takes time to form an abscess and exhibit symptoms. Phlegmonous gastritis due to complications other than ESD followed a similar pattern. Diffuse phlegmonous gastritis progresses faster than the localized type and has more severe symptoms with a poorer prognosis. The diffuse type is also associated with a higher mortality rate (54\%) than the localized type $(10 \%)^{2}$

The typical symptoms of phlegmonous gastritis are abdominal pain, fever, chills, nausea, vomiting, and hematemesis. ${ }^{2}$ Epigastric pain and fever have also been reported in cases phleg-

Table 1. Reported cases of phlegmonous gastritis or perigastric abscess after endoscopic resection in the literature

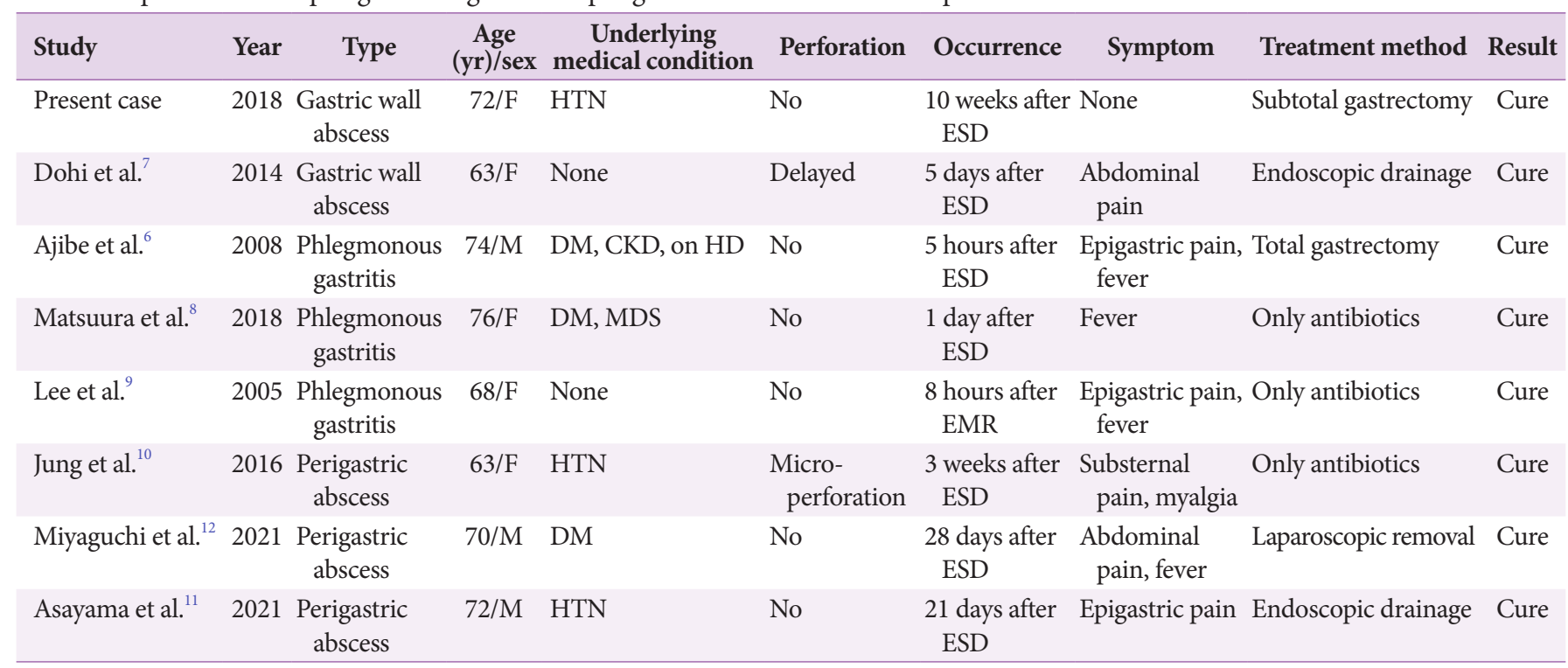

F, female; HTN, hypertension; ESD, endoscopic submucosal dissection; M, male; DM, diabetes mellitus; CKD, chronic kidney disease; HD, hemodialysis; MDS, myelodysplastic syndrome; EMR, endoscopic mucosal resection. 
monous gastritis after ESD. However, in our case, the patient had no symptoms, and the subepithelial lesion was only discovered as part of the routine follow-up after EGD. Regarding the management of phlegmonous gastritis, lower mortality rates are reported when surgery is performed. ${ }^{2}$

Among the cases we investigated, only two cases were administered antibiotics as treatment, surgical treatment was performed in three cases, and endoscopic drainage was performed in two cases. In our case, surgery was performed due to the possibility of cancer invasion.

Another characteristic feature of this case is the difference in CT findings of gastric wall abscess compared to that of other cases. In other cases, the abscess pattern was unilobular, and air-fluid levels were observed in the abscess lumen. ${ }^{13}$ In our case, the lesion was heterogenic, multilobular, and no gas was observed in the abscess lumen. The difference in CT findings may have been caused by the degree of abscess maturation. Differences in CT patterns based on abscess maturation should be considered when treating future gastric wall abscess cases.

In conclusion, the current report presents the first case of gastric wall abscess after ESD in Korea. As gastric wall abscess can also develop in immunocompetent patients with no symptoms, caution should be taken after ESD.

\section{Conflicts of Interest}

The authors have no potential conflicts of interest.

\section{Funding}

None.

\section{Acknowledgments}

The patient provided informed consent and agreed to publication of the images.

\section{Author Contributions}

Conceptualization: SHL, SRJ; Investigation: SJY; Project administration: SHL; Supervision: SHL; Visualization: SJY, JSY, HSL; Writing-original draft: SJY; Writing-review \& editing: JSY, SHL, HSL, SRJ.

\section{ORCID}

Seung Jung Yu
Sang Heon Lee Jun Sik Yoon

Hong Sub Lee

Sam Ryong Jee https://orcid.org/0000-0001-8210-0889 https://orcid.org/0000-0003-4328-3955 https://orcid.org/0000-0002-2962-0209 https://orcid.org/0000-0002-7928-1153

\section{REFERENCES}

1. Ono H, Yao K, Fujishiro M, et al. Guidelines for endoscopic submucosal dissection and endoscopic mucosal resection for early gastric cancer (second edition). Dig Endosc 2021;33:4-20.

2. Kim GY, Ward J, Henessey B, et al. Phlegmonous gastritis: case report and review. Gastrointest Endosc 2005;61:168-174.

3. Schultz MJ, van der Hulst RW, Tytgat GN. Acute phlegmonous gastritis. Gastrointest Endosc 1996;44:80-83.

4. Eley A, Richards HG. A case of localized gastric abscess. Br J Surg 1959;47:97-98.

5. Choong NWW, Levy MJ, Rajan E, et al. Intramural gastric abscess: case history and review. Gastrointest Endosc 2003;58:627-629.

6. Ajibe $\mathrm{H}$, Osawa $\mathrm{H}$, Yoshizawa $\mathrm{M}$, et al. Phlegmonous gastritis after endoscopic submucosal dissection for early gastric cancer. Therap Adv Gastroenterol 2008;1:91-95.

7. Dohi O, Dohi M, Inoue K, et al. Endoscopic transgastric drainage of a gastric wall abscess after endoscopic submucosal dissection. World J Gastroenterol 2014;20:1119-1122.

8. Matsuura K, Hiramatsu S, Taketani R, et al. Medical treatment of postendoscopic submucosal dissection phlegmonous gastritis in an elderly diabetic woman with myelodysplastic syndrome. Case Rep Gastrointest Med 2018;2018:8046817.

9. Lee BS, Kim SM, Seong JK, et al. Phlegmonous gastritis after endoscopic mucosal resection. Endoscopy 2005;37:490-493.

10. Jung JY, Kim JS, Kim BW, et al. Perigastric abscess as a complication of endoscopic submucosal dissection for early gastric cancer: first case report. Korean J Gastroenterol 2016;67:142-145.

11. Asayama N, Nagata S, Yukutake M, et al. A rare case of delayed perigastric abscess after curative resection of early gastric cancer by uncomplicated endoscopic submucosal dissection: successful treatment with endoscopic ultrasound-guided drainage. Intern Med 2021;60:1383-1387.

12. Miyaguchi K, Tashima T, Sugimoto K, et al. Intraperitoneal abscess as a postoperative complication of gastric endoscopic submucosal dissection. Intern Med 2021;60:2777-2781.

13. Asrani A, Novelline $\mathrm{R}$, Abujudeh $\mathrm{H}$, et al. Intramural gastric abscess--preoperative diagnosis with CT. Emerg Radiol 2007;14:253256. 